\title{
Properdin levels in human sepsis
}

\author{
Cordula M. Stover ${ }^{1}$, John McDonald ${ }^{2}$, Simon Byrne' ${ }^{1}$, David G. Lambert ${ }^{2}$ and Jonathan P. Thompson ${ }^{2}$ * \\ ' Department of Infection, Immunity and Inflammation, College of Medicine, Biological Sciences and Psychology, University of Leicester, Leicester, UK \\ 2 Department of Cardiovascular Sciences, Division of Anaesthesia, Critical Care and Pain Management, University of Leicester, Leicester, UK
}

\author{
Edited by: \\ Uday Kishore, Brunel University, UK \\ Reviewed by: \\ Michael Kirschfink, University of \\ Heidelberg, Germany \\ Arvind Sahu, National Centre for Cell \\ Science, India \\ *Correspondence: \\ Jonathan P. Thompson, Department \\ of Anaesthesia, Critical Care and Pain \\ Management, University Hospitals of \\ Leicester NHS Trust, Robert Kilpatrick \\ Clinical Sciences Building, Leicester \\ Royal Infirmary, Leicester LE2 \\ 7LX, UK \\ e-mail: jt23@le.ac.uk
}

Properdin is a normal serum protein that increases the production of complement activation products by binding C3b integral to convertase complexes and amplifying their activity at the site of activation. Thereby, it not only can aid in the resolution of infection but also contribute to tissue damage. In human sepsis, circulating complement $\mathrm{C} 3$ concentrations are decreased, though C3 is described as a positive acute phase reactant. However, properdin levels in human sepsis have not been reported. In this study, serum from 81 critically ill patients (predominately abdominal and respiratory sepsis) were analyzed for properdin levels at defined points of their stay in the intensive care unit (ICU) and compared with 61 age and sex-matched healthy volunteers. Properdin concentrations were significantly decreased in patients with sepsis on admission to ICU, but increased after clinical recovery to exceed levels observed in healthy volunteers. Properdin concentrations at ICU admission were decreased in non-survivors of sepsis compared to survivors, but this did not correlate with APACHE II score. However, pathologically low properdin levels $(<7 \mu \mathrm{g} / \mathrm{ml})$ were related to increased duration of treatment.

Keywords: sepsis, complement, properdin, intensive care, recovery

\section{INTRODUCTION}

Sepsis is a life threatening condition in which a systemic inflammatory reaction may progress to shock, hypoperfusion of lungs, kidneys, and intestines and via consumption of coagulation factors to intravascular coagulopathy. Cytokine storm, systemic macrophage activation, complement activation, and activation of the coagulation cascade are factors that drive the organism from a state of overinflammation to immune exhaustion. The complement system is primarily a humoral, hierarchically arranged, system in which activation of recognition molecules is translated to enzymatic activity of protein complexes, which cleave C3 and C5 to generate potent anaphylatoxins, C3a and C5a. The strength of activation also determines cell activities, such as phagocytosis, respiratory burst, and vascular leakage. The contribution of complement activation to tissue damage in septic conditions is thought to be so significant that attempts are made to develop therapeutic blockers in order to rein in its activity (1). However, because complement operates over a wide range of biological functions in health and disease, choosing the window of opportunity in a polymorphic system (2) is likely to be very difficult. In experimental animal models of sepsis, however, treatment with anti-C5a or anti-C5aR antibodies in clear relation to the defined onset of sepsis has proven efficacious $(3,4)$.

The role of low levels of mannan binding lectin (MBL), one of the lectin pathway recognition molecules, as a single determinant in the outcome from severe shock is not clear; while studies link low MBL levels to an increased risk of developing the systemic inflammatory response syndrome, others assign a beneficial effect to low MBL levels because of the potentially reduced pro-inflammatory reaction (5). A prospective study of septic patients in comparison with healthy controls found depressed levels of complement C3 and elevated complement activation products $\mathrm{C} 3 \mathrm{a}$, Factor $\mathrm{Bb}$ and
C4d, during the first 2 days of intensive care treatment (6). The observed increase in C5a in septic samples is consistent with previous work analyzed by the Bayesian inference approach (6). However, in sepsis, significant amounts of C5aR are found in serum, most likely shed from neutrophils (7); properdin is produced by neutrophils (8). Properdin is the only amplifier of complement activation but there are no data on properdin levels in human sepsis. A genetic deficiency of properdin predisposes to death from meningococcal septicemia in man (9), and in mice, significant impairment of survival is observed in endotoxin shock (10).

The aim of this study was to investigate properdin concentrations in critically ill patients admitted to the intensive care unit (ICU) with sepsis, using serum samples taken during a recently published observational study (11).

\section{MATERIALS AND METHODS}

Patients admitted to ICU at Leicester Royal Infirmary with a diagnosis of sepsis, and healthy volunteers matched for age and sex were recruited, as previously described (11). Permission to measure properdin in these samples was granted by University Hospitals of Leicester NHS Trust (R\&D reference: UHL 11348 "Properdin and inflammatory biomarkers in sepsis"). Patients' samples were taken on Days 1 and 2 of ICU admission, and a further sample was obtained after clinical recovery from sepsis. Archived, anonymized serum samples from 81 patients $(40 \mathrm{~m} / 41 \mathrm{f} ; 19-87$ years $)$ and of 61 age and sex-matched volunteers ( $29 \mathrm{~m} / 32 \mathrm{f} ; 21-85$ years $)$ were analyzed. The origin of sepsis was as follows: abdominal $n=41$, respiratory $n=25$, neutropenic $n=5$, urosepsis $n=2$, and a small group of $n=8$ made up of line infection $(n=2)$, fasciitis $(n=4)$, postpartum sepsis $(n=1)$, and abscess $(n=1)$.

Properdin was determined from $1 / 5000$ serum sample dilutions using commercial kits (Hycult Biotech, Uden, The Netherlands), 
following the manufacturer's protocol. Concentrations from duplicate measurements were calculated from a standard curve set up in duplicate. Those below the lowest dilution of the standard curve (cut off $0.3 \mathrm{ng} / \mathrm{ml}$ ) were classed as deficient (zero).

\section{STATISTICAL ANALYSIS}

Data for properdin concentrations were normally distributed and were analyzed by an unpaired $t$-test between two groups. Duration of ICU or hospital stay was not normally distributed and was compared against properdin concentrations by non-parametric two-tailed Spearman correlation. $P$-values $<0.05$ were considered statistically significant.

\section{RESULTS AND DISCUSSION}

Samples were available for 81,66 , and 45 patients on Days 1, 2 and recovery, respectively. Clinical and other data have been reported previously (11). While consecutive levels were significantly lower at Days 1 and 2 of ICU admission compared to matched volunteers, levels increased significantly at clinical recovery (Table 1). Properdin levels did not correlate with survival time in those who succumbed within 1-3 days after admission to ICU. There was no relationship between properdin and total white cell or neutrophil counts. Properdin levels were significantly higher after

Table 1 | Properdin levels in sera of critically ill patients with sepsis admitted to ICU and a healthy control group.

\begin{tabular}{lcc}
\hline Group & Range $(\boldsymbol{\mu g} / \mathbf{m l})$ & Mean \pm SD \\
\hline Day 1 $(n=81)$ & $0-38.8$ & $9.0 \pm 7.6$ \\
Day 2 $(n=66)$ & $0-30.9$ & $8.9 \pm 6.9$ \\
Clinically recovered $(n=45)$ & $2.4-51.2$ & $22.9 \pm 11.1^{\mathrm{a}, \mathrm{b}}$ \\
Volunteers $(n=61)$ & $7.6-34.10$ & $18.4 \pm 5.5^{\mathrm{c}}$ \\
Day 1, survivors $(n=60)$ & $0-38.8$ & $9.8 \pm 8.1$ \\
Day 1, non-survivors $(n=21)$ & $0-21.2$ & $6.8 \pm 5.2$ \\
\hline
\end{tabular}

The time point of clinical recovery from sepsis was variable.

${ }^{a} p<0.0001$ against Days 1 and 2 .

${ }^{b} p<0.0008$ against volunteers.

${ }^{c} p<0.0001$ against Days 1 or 2; Day 1 vs Day 2 n.s. clinical recovery compared to healthy volunteers. At the timepoint of clinical recovery, however, there was still biochemical evidence of inflammation (CRP and IL-8 concentrations had not completely normalized; increased leukocyte, neutrophil and platelet counts compared to the control group) (11). Properdin levels in the healthy volunteers of European background analyzed as part of this study were higher than those reported in a recent study for healthy South East Asians (12).

The overall 30-day mortality in patients was 21/81 (25.9\%). For four patients, in whom there (reproducibly) was no antigenically detectable properdin in their initial serum samples, no subsequent sample existed to exclude a genetic deficiency. But, as properdin is an $\mathrm{x}$-chromosome linked gene and a primary deficiency uncommon, the possibility of a gene defect does not appear likely in the case of the two female patients. When comparing properdin levels determined for samples obtained on day 1 of admission to ICU between survivors and non-survivors, properdin appeared to be lower in non-survivors but this did not reach statistical significance (Table 1).

Properdin levels determined on day 1 of ICU admission did not relate to APACHE or SOFA scores (data not shown) or duration of stay in ICU or in hospital, but for the whole group, the ICU stay correlated significantly with hospital stay (80 pairs analyzed, $r=0.6, p<0.0001)$. In other studies, depressed C3 levels found in sepsis were not related to mortality or complications such as pneumonia and hemorrhage (13), but decreased C3 levels were linked to increased hospital stay (14). Therefore, a subgroup analysis was performed for very low properdin levels. These were defined as $<7 \mu \mathrm{g} / \mathrm{ml}$, the lower end of the normal range established in this study. Pathologically low properdin levels on Days 1 and 2 negatively correlated with the duration of intensive care treatment and overall hospitalization, respectively (Figures 1A,B).

There are two possible interpretations for these findings: (i) the decline in properdin levels is a descriptor of severity of sepsis due to its ability to bind to LPS of different pathogens (15) or to damaged cells (16) and (ii) properdin is involved in the pathophysiological mechanisms of sepsis.

Recent data indicate that the likely determinants of outcome from sepsis are the cell phenotypes, which emanate from this condition in dependence of the level of complement components.

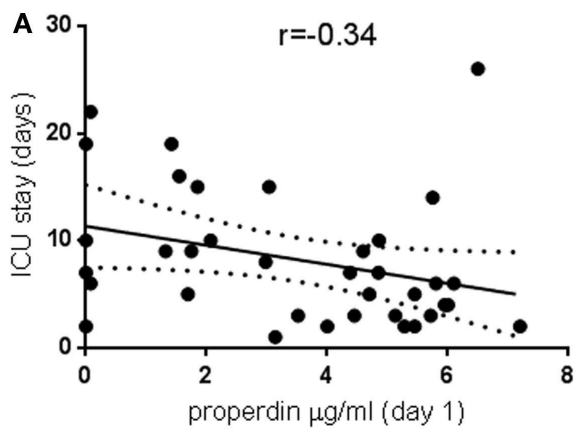

FIGURE 1 | Pathologically low properdin levels in the critically ill and the relationship to treatment duration. Properdin levels lower than $7 \mu \mathrm{g} / \mathrm{ml}$, the lowest of the normal range established in this study, were plotted against duration of intensive care treatment

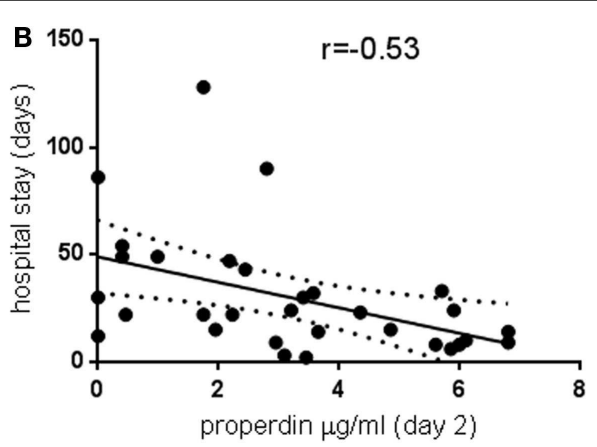

(A) 36 pairs, $p<0.05$, and total duration of hospitalization (B) 31 pairs, $p<0.005$. Spearman correlation coefficients are indicated; regression lines and $95 \%$ confidence bands of the best-fit line are shown. 
Depressed serum C3 levels have been linked to the emergence in the critically ill of an immune suppressive $\mathrm{T}_{\text {reg }}$ population (14). So, altered complement levels may be a mirror of changing cell activities selected during the septic immune response (17). Alternatively, a decline in circulating properdin levels may signify increased complement usage in septic end organs (18) or on inflamed endothelium (19).

\section{ACKNOWLEDGMENTS}

Funding for the study during which these samples were obtained was provided by the Anaesthesia/AAGBI Small Project Grant (2009). The data reported in this study were funded entirely by Departmental Funds.

\section{REFERENCES}

1. Markiewski M, DeAngelis D, Lambris J. Complexity of complement activation in sepsis. J Cell Mol Med (2008) 12:2245-54. doi:10.1111/j.1582-4934.2008. 00504.x

2. Heurich M, Martínez-Barricarte R, Francis NJ, Roberts DL, Rodríguez de Córdoba S, Morgan BP, et al. Common polymorphisms in C3, factor B, and factor $\mathrm{H}$ collaborate to determine systemic complement activity and disease risk. Proc Natl Acad Sci U S A (2011) 108:8761-6. doi:10.1073/pnas.1019338108

3. Riedemann NC, Guo RF, Neff TA, Laudes IJ, Keller KA, Sarma VJ, et al. Increased C5a receptor expression in sepsis. J Clin Invest (2002) 110:101-8. doi:10.1172/JCI200215409

4. Ward PA. The harmful role of C5a on innate immunity in sepsis. J Innate Immun (2010) 2:439-45. doi:10.1159/000317194

5. De Pascale G, Cutuli SL, Pennisi MA, Antonelli M. The role of mannose-binding lectin in severe sepsis and septic shock. Mediators Inflamm (2013) 2013:625803. doi:10.1155/2013/625803

6. Younger JG, Bracho DO, Chung-Esaki HM, Lee M, Rana GK, Sen A, et al. Complement activation in emergency department patients with severe sepsis. Acad Emerg Med (2010) 17:353-9. doi:10.1111/j.1553-2712.2010.00713.x

7. Unnewehr H, Rittirsch D, Sarma JV, Zetoune F, Flierl MA, Perl M, et al. Changes and regulation of the $\mathrm{C} 5 \mathrm{a}$ receptor on neutrophils during septic shock in humans. J Immunol (2013) 190:4215-25. doi:10.4049/jimmunol.1200534

8. Wirthmueller U, Dewald B, Thelen M, Schäfer MK, Stover C, Whaley K, et al. Properdin, a positive regulator of complement activation, is released from secondary granules of stimulated peripheral blood neutrophils. J Immunol (1997) 158:4444-51.

9. Späth PJ, Sjöholm AG, Fredrikson GN, Misiano G, Scherz R, Schaad UB, et al. Properdin deficiency in a large Swiss family: identification of a stop codon in the properdin gene, and association of meningococcal disease with lack of the IgG2 allotype marker G2m(n). Clin Exp Immunol (1999) 118:278-84. doi:10.1046/j.1365-2249.1999.01056.x

10. Ivanovska ND, Dimitrova PA, Luckett JC, El-Rachkidy Lonnen R, Schwaeble WJ, Stover CM. Properdin deficiency in murine models of nonseptic shock. J Immunol (2008) 180:6962-9. doi:10.4049/jimmunol.180.10.6962
11. Thompson JP, Serrano-Gomez A, McDonald J, Ladak N, Bowrey S, Lambert DG. The Nociceptin/Orphanin FQ system is modulated in patients admitted to ICU with sepsis and after cardiopulmonary bypass. PLoS One (2013) 8:e76682. doi:10.1371/journal.pone.0076682

12. Somani R, Richardson VR, Standeven KF, Grant PJ, Carter AM. Elevated properdin and enhanced complement activation in first-degree relatives of South Asian subjects with type 2 diabetes. Diabetes Care (2012) 35:894-9. doi:10.2337/dc11-1483

13. Ren J, Zhao Y, Yuan Y, Han G, Li W, Huang Q, et al. Complement depletion deteriorates clinical outcomes of severe abdominal sepsis: a conspirator of infection and coagulopathy in crime? PLoS One (2012) 7:e47095. doi:10.1371/journal.pone.0047095

14. Yuan Y, Yan D, Han G, Gu G, Ren J. Complement C3 depletion links to the expansion of regulatory $\mathrm{T}$ cells and compromises $\mathrm{T}$-cell immunity in human abdominal sepsis: a prospective study. J Crit Care (2013) 28:1032-8. doi:10.1016/j.jcrc.2013.09.007

15. Kimura Y, Miwa T, Zhou L, Song WC. Activator-specific requirement of properdin in the initiation and amplification of the alternative pathway complement. Blood (2008) 111:732-40. doi:10.1182/blood-2007-05-089821

16. Kemper C, Atkinson JP, Hourcade DE. Properdin: emerging roles of a patternrecognition molecule. Annu Rev Immunol (2010) 28:131-55. doi:10.1146/ annurev-immunol-030409-101250

17. Boomer JS, Green JM, Hotchkiss RS. The changing immune system in sepsis: is individualized immune-modulatory therapy the answer? Virulence (2014) 5:45-56. doi:10.4161/viru.26516

18. Nuytinck JK, Goris RJ, Weerts JG, Schillings PH, Stekhoven JH. Acute generalized microvascular injury by activated complement and hypoxia: the basis of the adult respiratory distress syndrome and multiple organ failure? Br J Exp Pathol (1986) 67:537-48.

19. Fox ED, Heffernan DS, Cioffi WG, Reichner JS. Neutrophils from critically ill septic patients mediate profound loss of endothelial barrier integrity. Crit Care (2013) 17:R226. doi:10.1186/cc13049

Conflict of Interest Statement: The authors declare that the research was conducted in the absence of any commercial or financial relationships that could be construed as a potential conflict of interest.

Received: 03 November 2014; accepted: 12 January 2015; published online: 02 February 2015.

Citation: Stover CM, McDonald J, Byrne S, Lambert DG and Thompson JP (2015) Properdin levels in human sepsis. Front. Immunol. 6:24. doi: 10.3389/fimmu.2015.00024

This article was submitted to Molecular Innate Immunity, a section of the journal Frontiers in Immunology.

Copyright (C) 2015 Stover, McDonald, Byrne, Lambert and Thompson. This is an openaccess article distributed under the terms of the Creative Commons Attribution License (CC BY). The use, distribution or reproduction in other forums is permitted, provided the original author(s) or licensor are credited and that the original publication in this journal is cited, in accordance with accepted academic practice. No use, distribution or reproduction is permitted which does not comply with these terms. 